\title{
The association of Crohn's disease with celiac disease
}

\author{
Iliass Charif, Mohamed El Abkari, Adil Ibrahimi, Mounia El Yousfi \\ Department of Hepatology and Gastroenterology, Hassan II University Hospital, Fes, Morocco \\ Email: charifiliass82@hotmail.com
}

Received 11 July 2012; revised 13 August 2012; accepted 26 August 2012

\begin{abstract}
We report two cases of a man and a woman whose association of Crohn's disease (CRD) and celiac disease (CD) was evident and significant. The characteristic of our patients was the young age of discovery, and the localization of the CRD which was different in the two cases: colic localization in the woman and ileal stenosing in the man. The diagnosis of MCO was confirmed by the histological study of the jejunal biopsies in both cases and by serologies (anti-gliadines Antibody) in one case. Through these two observations, we insist on the singularity of the association of the celiac disease to the Crohn's disease and on the complex etiopathogenesis of CRD which could have common points with that of $C D$. This fact can help us to understand more the two diseases and thereafter master their management.
\end{abstract}

Keywords: Crohn's Disease; Celiac Disease

\section{INTRODUCTION}

The association of celiac disease to Crohn's disease is exceptionally described. It has been reported mainly in the form of exceptional cases in the literature [1-3]. However, its frequency is probably underestimated because of the often latent character of the celiac disease. This association represents only $20 \%$ of associations with inflammatory bowel disease (IBD) [1,3]. We report two cases collected at the University Hospital Hassan II of Fez in Morocco.

\section{OBSERVATIONS}

\subsection{Observation No. 1}

Mrs. H.A. is 23 years old, non smoker, have been presenting intermittently for 10 years a chronic diarrhea associated with a dysentery and alteration of general condition. On physical examination, the patient was in a very poor condition (weight $=36 \mathrm{~kg}$ ), with hemodynamic instability, and clinical symptoms of anemia. Laboratory tests showed microcytic hypochromic anemia at $11.5 \mathrm{~g} / \mathrm{dl}$, a sedimentation rate at $31 \mathrm{~mm}$ in the first hour and hypoproteinemia to $58 \mathrm{~g} / \mathrm{l}$. Abdominal ultrasound showed a peritoneal effusion of middle abundance without evident digestive thickeness. The ileo-colonoscopy showed lesions suggestive of Crohn's disease with congestive non ulcerated colonic mucosa distributed with intervals of healthy mucosa, whereas the caecum and ileum appeared macroscopically normal. Histological study of colonic biopsies showed a lot of elements suggestive of IBD (abraded surface epithelium with elongation and vertical integration of the crypts, dense polymorphous inflammatory infiltrate in the chorion without crypt abscesses or granulomas and the presence of subacute ileitis with superficial ulceration). Oesogastroduodenal endoscopy showed a crenellated duodenal mucosa with mosaic feature. Histological examination of duodenal biopsies found a celiac disease type 4 according to the classification of Marsh with completely flattened villi, an inflammatory infiltrate in the lamina propria and lymphocytic exocytosis at 56 lymphocytes/100 enterocytes. So, all these examinations have confirmed the association of celiac disease with Crohn's disease in this first case.

Because the Crohn's disease was considered inactive according to the Best score, the decision was to start gluten-free diet and complete the management next according to the evolution of the disease. The patient was readmitted next in medical consultation with good clinical response. Gluten-free diet was maintaining and the crohn's disease was still in remission.

\subsection{Observation No. 2}

Mr. MM is 55 years old, had surgery two years ago for appendicular plastron (appendectomy not done), since there he has suffered from watery diarrhea with pain in the right iliac fossa and conservation of the general condition. Abdominal examination found a pain at the right iliac fossa. Laboratory tests showed an isolated inflammatory syndrome. Abdominopelvic ultrasound showed an inflammatory thickeness of the digestive tract in the right iliac fossa and ascites of low abundance. The CT enteroclysis showed that this digestive thickeness was at the expense of the last ileal loop. At the ileo-colonoscopy, 
ileal mucosa was ulcerated and congestive five centimeters long after the ileocecal valve with ileal impassable stricture. The colon was macroscopically normal. Histological examination of ileal biopsies showed lesions of Crohn's disease in acute exacerbation with the presence of an inflammatory infiltrate of the chorion, granules rich in neutrophils and cryptic abnormalities in type of duplication. During his hospitalization, the patient had epigastric pain justifying an oesogastroduodenal endoscopy which was normal but the histological study of jejunal biopsies found a celiac disease (atrophic mucosa with flattening of the villi, lymphocytosis > 40/100 enterocytes). The antigliadin antibodies were positive (IgA and IgG), whereas the anti-endomysium and anti-transglutaminase antibodies were negative.

So, our patient presented a combination of stenosing active Crohn's disease interesting the small bowel (Best score at 237) and celiac disease. The search for an associated thyroiditis was negative (TSHus was at $0.60 \mu \mathrm{UI} /$ $\mathrm{ml}$ ). The patient was put on oral corticosteroid therapy at a dose of $1 \mathrm{mg} / \mathrm{Kg} /$ day and gluten-free diet. The evolution was marked by a durable good clinical response after 12 months of follow-up; degression of corticosteroids was started after one month of treatment at full dose.

\section{DISCUSSION}

The association of celiac disease with IBD is rare. However, during the last decade, the prevalence of IBD especially CRD in patients with celiac disease has increased continuously; this prevalence is 5 to 10 times more frequent than in the general population $[1,4,5]$. Unlike our two patients with different ages and sexes, the association of celiac disease and Crohn's disease affects most often the young male adult $[1,4]$. We don't have an explanation to this epidemiological observation. And several studies are ongoing for giving an explanation for this fact. The etiopathogenesis of this association is not yet clear and several hypotheses have been advanced but none has been confirmed $[2,4,6]$ :

1) The rarity of similar cases reported in the literature may argue for an incidental association;

2) A particular genetic predisposition that may explain this association has been suggested by Cottone et al. [7] who described three cases in three different families of Sicily;

3) A hyper-intestinal permeability to antigens observed in celiac disease may promote immunological phenomena responsible for Crohn's disease [8].

The clinical picture of this association is dominated by diarrhea that is frequent in the two diseases. The characteristics of this diarrhea lead to one or the other of the two diseases. In celiac disease, diarrhea is fat and abundant, whereas diarrhea containing blood and mucous indicates IBD. The presence of anoperineal lesions directs towards the diagnosis of Crohn's disease [9]. In celiac disease, the lack of response to the RSG can be related to associated pathology and perform a comprehensive assessment of the digestive tract [10]. Karoui et al. [2] reported a case of an association of asymptomatic celiac disease with Crohn's disease in a 24 year old woman who was diagnosed with Crohn's disease for many years; the diagnosis of celiac disease was made by immunological tests and duodenal biopsies. Indeed, the presence of anti-gliadin antibodies and anti-endomysium antibodies allowed confirming the diagnosis of celiac disease. The essential benefit of the use of serological tests is to confirm the diagnosis of clinically latent celiac disease (pauci-symptomatic or subclinical). These silent forms are in fact the most common, which underestimates the prevalence of the disease [2], and make the diagnosis and the management of the patients suffered from both of these two diseases more difficult. A lot of common points seem unify these two diseases. Understanding these points can lead us to master the complexe etiopathogenesis of this association of crohn's disease with celiac disease and consequently make progress in the treatment of both of theme.

\section{CONCLUSION}

The association of Crohn's disease with celiac disease is rare and must be sought through comprehensive digestive tests in the event of warning signs or therapeutic resistance to one of these two conditions. Insisting on the importance of this association will open new fields of research concerning the etiopathogenesis of both of these diseases and will enable us to control them more and more.

\section{REFERENCES}

[1] Kitis, G., et al. (1980) Association of coeliac disease and inflammatory bowel disease. Gut, 21, 636-641. doi:10.1136/gut.21.7.636

[2] Karoui, S., et al. (2000) Association of asymptomatic celiac disease and Crohn's disease. Annals of Internal Medicine (Paris), 151, 411-412.

[3] Gillberg, R., Dotevall, G. and Ahren, C. (1982) Chronic inflammatory bowel disease in patients with coeliac disease. Scandinavian Journal of Gastroenterology, 17, 491496. doi:10.3109/00365528209182237

[4] Chakraborty, A., et al. (2003) Coeliac disease and Crohn's disease: An association not to be forgotten. Hospital Medicine, 64, 684-685.

[5] Leeds, J.S., et al. (2007) Is there an association between coeliac disease and inflammatory bowel diseases? A study of relative prevalence in comparison with population controls. Scandinavian Journal of Gastroenterology, 42, 1214-1220. doi:10.1080/00365520701365112 
[6] Snook, J.A., de Silva, H.J. and Jewell, D.P. (1989) The association of autoimmune disorders with inflammatory bowel disease. The Quarterly Journal of Medicine, 72, 835-840.

[7] Cottone, M., et al. (1989) Familial association of Crohn's and coeliac diseases. Lancet, 2, 338. doi:10.1016/S0140-6736(89)90527-8

[8] Ventura, A., Magazzu, G. and Greco, L. (1999) Duration of exposure to gluten and risk for autoimmune disorders in patients with celiac disease. SIGEP study group for autoimmune disorders in celiac disease. Gastroenterology, 117, 297-303. doi:10.1053/gast.1999.0029900297

[9] Curtis, W.D., Schuman, B.M. and Griffin, J.W., Jr. (1992) Association of gluten-sensitive enteropathy and Crohn's colitis. American Journal of Gastroenterology, 87, 16341637.

[10] Cheikh, I., et al. (2003) Association of celiac disease and Crohn's disease. A case report. Revue de Médecine Interne, 24, 755-756. doi:10.1016/S0248-8663(03)00246-7 